\title{
Unexpected doxorubicin-induced cardiomyopathy in sisters
}

\author{
Arlene A. Gayle $\cdot$ Kamakshi Sachidanandam • \\ Jill Kolesar · H. Ian Robins
}

Received: 13 July 2011/Accepted: 26 September 2011/Published online: 8 November 2011

(C) The Japan Society of Clinical Oncology 2011

\begin{abstract}
We report the details of two unexpected cases of doxorubicin (DOX)-induced cardiac toxicity in two sisters receiving adjuvant breast cancer therapy. One at age 38 developed a cardiomyopathy resulting in cardiac transplantation 6.5 years after receiving a relatively low total dose of DOX, i.e., $280 \mathrm{mg} / \mathrm{m}^{2}$. The second at age 60 also developed a severe cardiomyopathy (i.e., a ventricular ejection fraction of 22\%) 6 years after receiving a total dose of DOX of only $158 \mathrm{mg} / \mathrm{m}^{2}$. Twelve single nucleotide polymorphisms previously associated with doxorubicin cardiotoxicity were assessed by pyrosequencing. None of the genotypes associated with an increased risk of doxorubicin-induced cardiotoxicity were identified in these two patients.
\end{abstract}

Keywords Doxorubicin · Cardiotoxicity · Polymorphism

\section{Introduction}

The anthracycline anti-cancer agent doxorubicin (DOX) has long been recognized to induce a dose-limiting cardiotoxicity. The average incidence of $5.1 \%$ at $400 \mathrm{mg} / \mathrm{m}^{2}$ increases above $500 \mathrm{mg} / \mathrm{m}^{2}$, although with substantial individual variations [1]. Many studies support the theory that mitochondria are the primary target of doxorubicin-induced oxidative stress both acutely and long term [2]. Anthracycline cardiotoxicity (ACT) may be acute or chronic. Acute ACT occurs during treatment, often immediately after the first

\footnotetext{
A. A. Gayle $(\bowtie) \cdot$ K. Sachidanandam · J. Kolesar · H. I. Robins Department Hematology and Oncology, University of Wisconsin Hospital and Clinics, University of Wisconsin Carbone Comprehensive Cancer Center, K6/510 CSC, 600 Highland Ave, Madison, WI 53792, USA

e-mail: hirobins@facstaff.wisc.edu
}

dose, and manifests itself predominantly in the form of arrhythmias; rarely pericarditis, myocarditis and acute left ventricular failure are observed. Chronic ACT presents within 1 year after anthracycline administration as congestive heart failure. In addition, recent studies postulate a distinct late-onset cardiotoxicity, which develops after years or even decades of asymptomatic survival [3].

Transgenic mouse models have demonstrated a genetic etiology in the development of chronic ACT [3]. The overexpression of the multiple drug-resistant gene, MDR1, protects the heart from the toxic effect of doxorubicin [4]. In humans, the presence of a genetic component is suggested by the wide variation and individual sensitivities to anthracyclines [5]. In the context of investigating $\mathrm{NAD}(\mathrm{P}) \mathrm{H}$ oxidase and multidrug resistance protein genetic polymorphisms in association with doxorubicin-induced cardiotoxicity (with data derived from a multi-center phase II trial), Wojnowski et al. [3] concluded that "genetic variants in doxorubicin transport and free radical metabolism may modulate individual risk to develop ACT." In this regard, it was concluded that the molecular basis of adriamycin (AQ)-dependent development of cardiotoxicity is still far from being clear [6].

Relative to this background, we report two unexpected cases (on the basis of low DOX exposure) of severe presumed doxorubicin (DOX)-induced cardiac toxicity in two sisters receiving adjuvant breast cancer therapy. Included in the report is an analysis of 12 single nucleotide polymorphisms (SNPs) previously reported to have an association with doxorubicin cardiotoxicity.

\section{Case reports}

Sister no. 1 was diagnosed in August 1995 at age 32 with a stage IIIa infiltrating ductal carcinoma involving the left 
breast. The tumor was $2.3 \times 1.5 \mathrm{~cm}$, ER/PR positive, HER2 negative, poorly differentiated Bloom-Richardson grade 3 and extending to the margins. She had $4 / 20$ nodes positive. She underwent a lumpectomy with axillary lymph node dissection followed by chemotherapy and then radiation therapy to the left breast and axilla. She received chemotherapy on the ECOG C9344 protocol and was randomized to receive DOX with cyclophosphamide followed by paclitaxel, which was completed on 30 January 1996. Her total dose of was DOX $288 \mathrm{mg} / \mathrm{m}^{2}$. She was maintained on adjuvant tamoxifen therapy for approximately 5 years. In July 2002 she developed congestive heart failure and was found to have a dilated cardiomyopathy and ejection fraction of $10-15 \%$, and severe diastolic dysfunction. She had an automatic implantable cardioverter defibrillator (AICD) placed on 3 September 2002 for non-sustained ventricular tachycardia. The patient underwent an orthotopic heart transplant 8 January 2003. Prior to her breast cancer diagnosis, she had no cardiac history. She did not have a baseline echocardiogram prior to starting chemotherapy on 6 September 1995, but she had a 12-lead electrocardiogram, which was normal. The pathology report showed no radiation effect as well as "myocardium with focal patchy interstitial fibrosis consistent with cardiomyopathy."

Sister no. 2 was diagnosed at age 44 years (19 March 1999 ) with a left breast ER negative, PR $3+$ positive and HER-2 negative, poorly differentiated, grade $3,1.2 \times 1.1$ $\mathrm{cm}$ infiltrating ductal carcinoma with an intraductal cribriform component and all ten lymph nodes negative. She underwent a left segmental mastectomy and axillary lymph node dissection followed by chemotherapy with DOX and cyclophosphamide for two of four cycles followed by left breast irradiation. She was then given adjuvant tamoxifen. Chemotherapy was recommended in view of her high-risk features. The patient had diabetes insipidus secondary to a resected schwannoma and noted an increase in her DDAVP requirements while on chemotherapy; therefore, she discontinued the chemotherapy after two cycles. Her total dose of DOX was $158 \mathrm{mg} / \mathrm{m}^{2}$ completed 21 May 1999. She developed congestive heart failure in 2005. An echocardiogram done on 28 April 2005 confirmed severe left ventricular systolic function with severe diffuse global left ventricular hypokinesia. The quantitative left ventricular ejection fraction was $22 \%$. The right ventricular systolic function was also depressed. Prior to her breast cancer diagnosis, she had no cardiac history.

The genotyping analysis was performed in the Analytical Instrumentation Laboratory for Pharmacokinetics, Pharmacodynamics and Pharmacogenetics, University of Wisconsin Comprehensive Cancer Center, Madison, WI, USA). The patients' consent to participate in this study was obtained in compliance with existing institutional review board requirements.
Sample collection

Ten milliliters of whole blood was collected from each patient on a return clinical visit after hospitalization. The samples were collected in DNA Paxgene tubes and shipped at ambient temperature to the reference laboratory for further processing.

\section{Extraction of genomic DNA}

The genomic DNA was extracted from the patients' whole blood using the PAXgene DNA extraction kit as per the manufacturer's recommendations and quantified using a NanoDrop ND-1000 spectrophotometer (ThermoFisher Scientific, Waltham, MA, USA) with yields of 133.4 and $173.5 \mathrm{ng} / \mu \mathrm{l}$ for sister no. 1 and sister no. 2 , respectively. The purified genomic DNA was stored at $4{ }^{\circ} \mathrm{C}$ for pending analysis.

The genomic analysis

SNPs were evaluated for both patients in the following genes-ABCB1 (rs1045642, rs1128503 and rs2032582) [7], NADPH oxidase (rs4673, rs1883112 and rs13058338), CBR1 (rs1143663 and rs41557318) and CBR3 (rs1056892 and rs2835285). PCR was used to amplify the regions of the genomic DNA containing the selected SNPs in the patient and control samples (genomic DNA from wild-type A549 lung cancer cells). The final PCR reaction mixture contained forward and reverse primers (10-25 pmol each; one of the primers is biotinylated at the $5^{\prime}$ end), template DNA (250 ng), $1 \times$ PCR master mix (Promega, Madison Wisconsin, WI, USA) and nuclease-free water to a final volume of $25 \mu$ l. The primers (sequences available upon request) were designed using the Pyromark Assay Design 2.0 software (Qiagen, Valencia, CA, USA) and synthesized by Integrated DNA Technologies (Coralville, IA, USA) with either the forward or the reverse primer biotinylated at the $5^{\prime}$ end. The PCR was performed in an MJ Research PTC-100 or PTC-200 (Waltham) thermocycler with the following settings $-95^{\circ} \mathrm{C}$ for $5 \mathrm{~min}, 50-55$ cycles of $95^{\circ} \mathrm{C}$ for $30 \mathrm{~s}, 52-59^{\circ} \mathrm{C}$ for $30 \mathrm{~s}$ and $72^{\circ} \mathrm{C}$ for $30 \mathrm{~s}$, followed by $72^{\circ} \mathrm{C}$ for 5 min with storage at $4^{\circ} \mathrm{C}$ post cycle. To confirm proper amplicon length, $5 \mu \mathrm{l}$ of each PCR product was subjected to electrophoresis on a $2 \%$ agarose gel containing $0.4 \mu \mathrm{g} / \mathrm{ml}$ of ethidium bromide and compared with a pGEM DNA ladder (Promega). Pyrosequencing analysis was performed to detect the presence or absence of SNPs. Briefly, the biotinylated PCR product was immobilized on streptavidin-coated Sepharose beads (GE Healthcare, Piscataway, NJ, USA) and washed with $70 \%$ ethanol, $10 \mathrm{mM}$ $\mathrm{NaOH}$ and $200 \mathrm{mM}$ Tris-acetate, $\mathrm{pH}$ 7.6, resulting in single-stranded DNA bound to Sepharose beads. The beads 
were captured onto probes by vacuum and released into respective sample wells containing the specific sequence primer. The single-stranded DNA was annealed to the sequence primer by heating and cooling, and subsequently analyzed using PyroGold reagents (Qiagen, Valencia, CA, USA) on a PSQ96 MD pyrosequencer (Qiagen).

\section{Genotyping analysis for identifying selected SNPs}

On the basis of a review of the literature, several candidate SNPs were selected for analysis as a result of their respective roles in either the pharmacokinetics, clinical response or cardiotoxicity induced by doxorubicin therapy $[2,3,8-13]$. The SNPs are located in specific genes that encode for the following proteins: ATP-binding cassette sub-family B member 1 (ABCB1 or MDR1), NADPH Oxidase and Carbonyl Reductases 1 and 3 (CBR1 and CBR3). A summary of the results of the selected SNPs are outlined in Table 1. Of the 12 SNPs assessed, neither patient had any genotype previously associated with doxorubicin cardiotoxicity.

\section{Discussion}

This case report evaluated two potential mechanisms of doxorubicin cardiotoxicity, i.e., the generation of reactive oxygen species (ROS) and transmembrane anthracycline transport. The two-electron reduction by enzymes such as aldo-keto reductases and carbonyl reductases, and the generation of ROS are thought to cause of ACT. Thus, SNPs in NADPH oxidase and carbonyl reductases 1 and 3 that are associated with ACT in other reports were evaluated [3]. Additionally, SNPs in ABCB1, one of the major transporters of doxorubicin, were also evaluated. However, neither subject had a genotype previously reported to be associated with ACT.

One explanation for the lack of association between these patient's genotypes and ACT is that other known or unknown genotypes are responsible for the observed ACT. While we did not evaluate every polymorphism potentially associated with ACT toxicity, we did assess C242T (His72Tyr, rs4673) and the NCF4 variant rs1883112, which are two of the best studied of the known polymorphisms. The NCF4 gene encodes the p40phox subunit of the $\mathrm{NAD}(\mathrm{P}) \mathrm{H}$ oxidase, which is responsible for the downregulation of the enzyme and the CYBA gene codes for $\mathrm{p} 22$ phox gene. Individuals with the CYBA $\mathrm{T}$ allele have less enzyme activity. A large retrospective series including 1,697 subjects with NHL receiving doxorubicin and 109 developing ACT found these two SNPs to be associated with both acute and chronic ACT (3).

An additional explanation in unknown genetic polymorphisms may be responsible. When comparing these cases to the literature, some important differences emerge. First, the doxorubicin dose received by our subjects was $158-288 \mathrm{mg} / \mathrm{m}^{2}$, which is lower than that commonly associated with ACT, where $500 \mathrm{mg} / \mathrm{m}^{2}$ is associated with an approximately 6\% incidence of ACT (3). Second, both subjects had worse cardiotoxicity than expected with ejection fractions of $10-15$ and $22 \%$ compared to the usual

Table 1 Summary of genotyping results of the selected SNPs

\begin{tabular}{|c|c|c|c|c|c|c|}
\hline \multirow[t]{2}{*}{ Gene } & \multirow[t]{2}{*}{ SNP } & \multirow[t]{2}{*}{ Frequency in Caucasians ${ }^{\mathrm{a}, \mathrm{b}}$} & \multirow[t]{2}{*}{ SNP type ${ }^{c}$} & \multicolumn{2}{|c|}{ Genotype } & \multirow{2}{*}{$\begin{array}{l}\text { Increased risk } \\
\text { genotype }^{\mathrm{d}}\end{array}$} \\
\hline & & & & Case 1 & Case 2 & \\
\hline ABCB1 & rs1045642 & C/C 0.125, C/T 0.5, T/T 0.375 & Synonymous & $\mathrm{C} / \mathrm{C}$ & $\mathrm{C} / \mathrm{T}$ & $\mathrm{T} / \mathrm{T}$ \\
\hline ABCB 1 & rs2032582 & G/G 0.323, G/T 0.484, T/T 0.193 & Missense (S893A) & $\mathrm{G} / \mathrm{G}$ & $\mathrm{G} / \mathrm{T}$ & $\mathrm{T} / \mathrm{T}$ \\
\hline $\mathrm{ABCB} 1$ & rs1128503 & C/C $0.323, \mathrm{C} / \mathrm{T} 0.419, \mathrm{~T} / \mathrm{T} 0.258$ & Synonymous & $\mathrm{C} / \mathrm{C}$ & $\mathrm{C} / \mathrm{T}$ & $\mathrm{T} / \mathrm{T}$ \\
\hline NADPH oxidase & rs 4673 & C/C $0.458, \mathrm{C} / \mathrm{T} 0.417, \mathrm{~T} / \mathrm{T} 0.533$ & Missense (Y72H) & $\mathrm{C} / \mathrm{C}$ & $\mathrm{C} / \mathrm{C}$ & $\mathrm{C} / \mathrm{T}$ and $\mathrm{T} / \mathrm{T}$ \\
\hline NADPH oxidase & rs 13058338 & T/T 0.533, T/A 0.383, A/A 0.083 & Synonymous & $\mathrm{T} / \mathrm{T}$ & $\mathrm{T} / \mathrm{T}$ & $\mathrm{A} / \mathrm{A}$ and $\mathrm{A} / \mathrm{T}$ \\
\hline NADPH oxidase & rs1883112 & G/G 0.3, G/A 0.5, A/A 0.2 & Synonymous & $\mathrm{G} / \mathrm{G}$ & $\mathrm{G} / \mathrm{A}$ & $\mathrm{A} / \mathrm{A}$ \\
\hline CBR1 & rs1143663 & G/G 1.00 & Missense (V88I) & $\mathrm{G} / \mathrm{G}$ & $\mathrm{G} / \mathrm{G}$ & $\mathrm{A} / \mathrm{G}$ \\
\hline CBR1 & rs41557318 & C/C 0.957, C/T 0.043 & Missense (P131S) & $\mathrm{C} / \mathrm{C}$ & $\mathrm{C} / \mathrm{C}$ & $\mathrm{C} / \mathrm{T}$ \\
\hline CBR3 & rs2835285 & G/G 0.967, G/A 0.033 & Missense (V93I) & $\mathrm{G} / \mathrm{G}$ & $\mathrm{G} / \mathrm{G}$ & $\mathrm{G} / \mathrm{A}$ \\
\hline CBR3 & rs 4987121 & A/A $0.033, \mathrm{~A} / \mathrm{G} 0.433, \mathrm{G} / \mathrm{G} 0.053$ & Missense (V244M) & $\mathrm{A} / \mathrm{G}$ & $\mathrm{A} / \mathrm{G}$ & $\mathrm{A} / \mathrm{A}$ \\
\hline
\end{tabular}

SNP single nucleotide polymorphism, $A B C B 1$ ATP-binding cassette subfamily B member 1, NADPH oxidase nicotinamide adenine dinucleotide phosphate oxidase, $C B R$ carbonyl reductase

${ }^{a}$ Frequencies in Caucasians were obtained from the HapMap CEU database

b The wild-type genotype appears first, followed by the heterozygote and the variant

c A synonymous SNP is one in which there is no amino-acid change

${ }^{\mathrm{d}}$ Listed genotypes have been shown to be associated with an increased risk of doxorubicin-induced cardiotoxicity 
definition of a $50 \%$ reduction in ejection fraction, and finally, despite receiving lower than usual doses of doxorubicin, both subjects are long-term survivors of breast cancer. Taken together, these clinical features and the lack of association with the best studied SNPs associated with ACT suggests a different etiology of ACT in these patients and potentially unknown genetic factors.

In summary, we report two cases of severe doxorubicininduced cardiotoxicity in two sisters. The etiology of the ACT is unknown, but appears unlikely to be related to generation of ROS and transmembrane anthracycline transport. Finally, the authors believe that this case report highlights the potential utility of inquiring into unusual toxic consequences of chemotherapy in family members when interviewing new perspective oncology patients.

Conflict of interest The authors declare that they have no conflict of interest.

\section{References}

1. Gianni L, Herman E, Lipshultz S, Minotti G, Sarvazyan N, Sawyer D (2008) Anthracycline cardiotoxicity: from bench to bedside. J Clin Oncol 26(22):3777

2. Berthiaume JM, Wallace KB (2007) Adriamycin-induced oxidative mitochondrial cardiotoxicity. Cell Biol Toxicol 23(1): 15-25. doi:10.1007/s10565-006-0140-y

3. Wojnowski L, Kulle B, Schirmer M, Schluter G, Schmidt A, Rosenberger A, Vonhof S, Bickeboller H, Toliat MR, Suk EK, Tzvetkov M, Kruger A, Seifert S, Kloess M, Hahn H, Loeffler M, Nurnberg P, Pfreundschuh M, Trumper L, Brockmoller J, Hasenfuss $\mathrm{G}$ (2005) NAD(P)H oxidase and multidrug resistance protein genetic polymorphisms are associated with doxorubicininduced cardiotoxicity. Circulation 112(24):3754-3762. doi:10. 1161/CIRCULATIONAHA.105.576850
4. Dell'Acqua G, Polishchuck R, Fallon JT, Gordon JW (1999) Cardiac resistance to adriamycin in transgenic mice expressing a rat alpha-cardiac myosin heavy chain/human multiple drug resistance 1 fusion gene. Hum Gene Ther 10(8):1269-1279. doi: $10.1089 / 10430349950017950$

5. Deng S, Wojnowski L (2007) Genotyping the risk of anthracycline-induced cardiotoxicity. Cardiovasc Toxicol 7(2):129-134

6. Gille L, Nohl H (1997) Analyses of the molecular mechanism of adriamycin-induced cardiotoxicity. Free Radic Biol Med 23(5): 775-782 pii: S0891-5849(97)00025-7

7. Sylvester RK, Steen P, Tate JM, Mehta M, Petrich RJ, Berg A, Kolesar J (2011) Temozolomide-induced severe myelosuppression: analysis of clinically associated polymorphisms in two patients. Anticancer Drugs 22(1):104-110. doi:10.1097/CAD. 0b013e3283407e9f

8. Ambrosone CB, Ahn J, Singh KK, Rezaishiraz H, Furberg H, Sweeney C, Coles B, Trovato A (2005) Polymorphisms in genes related to oxidative stress (MPO, MnSOD, CAT) and survival after treatment for breast cancer. Cancer Res 65(3):1105-1111 pii: $65 / 3 / 1105$

9. Couture L, Nash JA, Turgeon J (2006) The ATP-binding cassette transporters and their implication in drug disposition: a special look at the heart. Pharmacol Rev 58(2):244-258. doi:10.1124/pr.58.2.7

10. Behnia K, Boroujerdi M (1999) Inhibition of aldo-keto reductases by phenobarbital alters metabolism, pharmacokinetics and toxicity of doxorubicin in rats. J Pharm Pharmacol 51(11):1275-1282

11. Forrest GL, Gonzalez B, Tseng W, Li X, Mann J (2000) Human carbonyl reductase overexpression in the heart advances the development of doxorubicin-induced cardiotoxicity in transgenic mice. Cancer Res 60(18):5158-5164

12. Slupe A, Williams B, Larson C, Lee LM, Primbs T, Bruesch AJ, Bjorklund C, Warner DL, Peloquin J, Shadle SE, Gambliel HA, Cusack BJ, Olson RD, Charlier HA (2005) Reduction of 13-deoxydoxorubicin and daunorubicinol anthraquinones by human carbonyl reductase. Cardiovasc Toxicol 5(4):365-376 pii: CT:5: 4:365

13. Olson LE, Bedja D, Alvey SJ, Cardounel AJ, Gabrielson KL, Reeves RH (2003) Protection from doxorubicin-induced cardiac toxicity in mice with a null allele of carbonyl reductase 1 . Cancer Res 63(20):6602-6606 